\title{
Evaluation of Optimum Room Entry Times for Radiation Therapists after High Energy Whole Pelvic Photon Treatments
}

\author{
Lavine Ho, Peter White, Edward Chan, Kim Chan, Janet Ng and Timothy Tam \\ Department of Health Technology and Informatics, The Hong Kong Polytechnic University, Hong Kong
}

\begin{abstract}
Evaluation of Optimum Room Entry Times for Radiation Therapists after High Energy Whole Pelvic Photon Treatments: Lavine Ho, et al. Department of Health Technology and Informatics, The Hong Kong Polytechnic University, Hong KongObjectives: Linear accelerators operating at or above $10 \mathrm{MV}$ produce neutrons by photonuclear reactions and induce activation in machine components, which are a source of potential exposure for radiation therapists. This study estimated gamma dose contributions to radiation therapists during high energy, whole pelvic, photon beam treatments and determined the optimum room entry times, in terms of safety of radiation therapists. Methods: Two types of technique (anteriorposterior opposing and 3-field technique) were studied. An Elekta Precise treatment system, operating up to 18 $\mathrm{MV}$, was investigated. Measurements with an area monitoring device (a Mini 900R radiation monitor) were performed, to calculate gamma dose rates around the radiotherapy facility. Measurements inside the treatment room were performed when the linear accelerator was in use. The doses received by radiation therapists were estimated, and optimum room entry times were determined. Results: The highest gamma dose rates were approximately $7 \mu \mathrm{Sv} / \mathrm{h}$ inside the treatment room, while the doses in the control room were close to background $(\sim 0 \mu \mathrm{Sv} / \mathrm{h})$ for all techniques. The highest personal dose received by radiation therapists was estimated at $5 \mathrm{mSv} / \mathrm{yr}$. To optimize protection, radiation therapists should wait for up to11 min after beam-off prior to room entry. Conclusions: The potential risks to radiation therapists with standard safety procedures were well below internationally recommended values, but risks could be further decreased by delaying room entry times. Dependent on the technique used, optimum entry times ranged between 7 to $11 \mathrm{~min}$. A balance between moderate treatment times versus reduction in measured equivalent doses should be
\end{abstract}

Received Aug 1, 2011; Accepted Nov 22, 2011

Published online in J-STAGE Feb 10, 2012

Correspondence to: P. White, Department of Health Technology and Informatics, The Hong Kong Polytechnic University, Hung Hom, Kowloon, Hong Kong (e-mail: htpwhite@polyu.edu.hk) considered.

(J Occup Health 2012; 54: 131-140)

Key words: High energy photons, Radiographers, Room entry times

High energy photon beams (greater than $10 \mathrm{MV}$ ) are routinely employed in clinical use for treatment of deep-seated tumors ${ }^{1,2)}$. However, they induce undesirable photonuclear and electronuclear reactions ${ }^{3)}$ that produce neutrons and radioisotopes. Neutron production increases with photon energy, and the induced radioactivity depends on the neutron radiation level ${ }^{3,4}$. This phenomenon induces potential exposure for radiation therapists due to neutron, gamma and beta radiations emitted from decay of activation products ${ }^{1,3)}$. As occupational doses are of concern, undesirable photoneutrons should be minimized and actions taken to reduce unnecessary exposure.

\section{Neutron production}

The minimum energy required to remove a neutron from a nucleus decreases with increase in target atomic number ${ }^{5)}$ and lies between 6 to $16 \mathrm{MeV}$ for nuclei heavier than carbon ${ }^{6}$. Linear accelerators generate high energy photon beams by accelerating electrons to 6-25 MV and then converting them to therapeutic $\mathrm{X}$-rays. For photon energies below $10 \mathrm{MV}$, minimal photonuclear reaction occurs, and neutron production is negligible ${ }^{5}$. Neutrons are also produced by photodisintegration processes. However, since these have fewer interactions with the nuclei of the accelerator head components ${ }^{7}$, photonuclear reactions are considered to be the main cause of neutron contamination in external beam radiotherapy.

Neutron production takes place inside machinery components of the linear accelerator head and the bodies of patients, as well as in the walls of the treatment room ${ }^{1-4,8-11)}$. The target, beam collimation systems and multi-leaf collimators (MLCs) are the major sources of neutron production where the photon 
fluence and the target atomic number are high $^{2,6,8,12)}$. In fact, the threshold of neutron production for some metals comprising components of the machine head have been identified: $6-8 \mathrm{MeV}$ for lead and tungsten, $13 \mathrm{MeV}$ for aluminium and iron and $10 \mathrm{MeV}$ for copper ${ }^{1,9)}$. For a treatment using a $15 \mathrm{MV}$ photon beam, the mean energy of secondary neutrons is 1-3 MeV, while the maximum is $7.6 \mathrm{MeV}^{7}$.

\section{Factors affecting neutron production}

A change in photon energy affects neutron production. A sixfold increase in the neutron dose was measured around two Siemens Mevatron 77 accelerators as the photon energy was increased from $15 \mathrm{MV}$ to $18 \mathrm{MV}^{13)}$. The accelerator type also affects neutron production, so different target materials and machinery components may be used to reduce neutron production $^{14)}$. Beam modifying accessories, such as physical and dynamic wedges, MLCs and shielding are used in routine clinical practice. These accessories consist of high atomic number materials, and as they lie in the path of the primary beam, interactions occur and produce neutrons ${ }^{2,3,14-17)}$.

Different treatment techniques also have an effect on induced radioactivity. Advanced radiotherapy techniques, like three-dimensional conformal radiotherapy (3DCRT) and intensity-modulated radiotherapy (IMRT), provide high conformity for treatment targets and, therefore, reduce unnecessary radiation doses to surrounding healthy tissues. However, longer beam-on times for the same dose, when compared to conventional techniques, is a compromise. For longer irradiation times, more secondary neutrons are generated, hence increasing the amount of induced radioactivity $^{18)}$. In addition, smaller field sizes used for IMRT and MLCs affect neutron production and scatter ${ }^{1,17-20)}$.

\section{Radioactivity induced by neutron interaction}

Neutrons are twenty times more biologically damaging than photons, as revealed by the larger radiation weighting factor of 20 recommended by both the National Council on Radiation Protection and Measurements (NCRP) and the International Commission on Radiological Protection (ICRP) ${ }^{21,22)}$. Even small doses of neutrons can cause severe biological effects. In addition to the effects of neutron dose deposit, neutrons with thermal and resonance energies can activate objects in the treatment room through neutron capture reaction ${ }^{1,4)}$, which contributes significantly to occupational exposure. Some activation products become radioactive and undergo a decay process to emit gamma and beta radiations.

Extensive studies have been carried out on radioactive isotopes that are commonly present in treatment rooms. The half lives of activated tungsten, aluminium, magnesium and copper are $23.7 \mathrm{~h}, 2.3 \mathrm{~min}$, $9.46 \mathrm{~min}$ and $9.7 \mathrm{~min}$, respectively ${ }^{4,23,24)}$. Also, particles in air can be activated, with the radiation intensity varying with beam energy ${ }^{25-27)}$.

\section{Induced activity as a main concern for estimating occupational exposure}

The half-life of a single neutron in air is several microseconds, but the radioactivity remains and is accumulated; it is still capable of being detected several days after the last emission of the beam ${ }^{3,4)}$. Only $7 \%$ of the estimated annual effective dose due to external exposure is caused by the neutron dose, while $54 \%$ of the dose is due to decay of activation products $^{7}$. Therefore, radioactivity induced by neutron interactions should be the main concern for estimating occupational exposure.

Exposure to residual radioactivity is a potential health risk associated with radiation therapists. Gamma radiation from induced activity will give fairly uniform irradiation of radiation therapists who enter the treatment room, while beta radiation dominantly irradiates the hands when the radiation therapists come into direct contact with wedge filters or other activated accessories ${ }^{13,27-29)}$. As radiation therapists are associated with repetitive workflow, the damaging effects of radiation can be accumulated, and thus, radiation hazards should be minimized. Numerous papers have focused on radiation protection of patients ${ }^{8,16,25)}$, but few have focused on radiation protection of staff from secondary neutron-induced radioactivity. The emphasis has been on patient dose or on workplace monitoring, where the calibration and use of reading devices and instrumentation were the main focus. The current study emphasized the equivalent dose to radiographers who operated medical linear accelerators. It is known that photon dose equivalents decrease exponentially with distance from the treatment fields but that neutron dose equivalents are independent of this, so the current study sought to investigate how significant would room entry times be in terms of the equivalent dose to staff. Therefore, the benefit of the current study was to minimize receipt of unnecessary doses in radiographers if similar treatment methods were used, as compared with other studies that related more to patients.

\section{Current practice in Hong Kong}

Research on safe room entry times is scarce, and there is no standard room entry time after high-energy treatments. Currently, the practice in Hong Kong of radiation therapists waiting before entry after high energy photon treatments is quite discrepant, and protocols vary from hospital to hospital, with some hospitals recommending a waiting time of $3 \mathrm{~min}$ 
for $15 \mathrm{MV}$ photon energy treatments, or $15 \mathrm{~min}$ for $18 \mathrm{MV}$ treatments, while another advocates early entry after only $1 \mathrm{~min}$.

Annual occupational doses of radiation therapists from induced activity may be reduced through time delay before entry into the treatment room after high energy photon beam treatments ${ }^{22,28,30)}$. With a view to achieve the "As Low As Reasonably Achievable" (ALARA) principle, a compromise should be made between patient throughput and occupational radiation safety.

\section{Aims of the Study \\ The aims of this study were to estimate the annual absorbed doses of radiation therapists who delivered high energy pelvic treatments, to recommend the opti- mum times of entry into the treatment rooms after such treatments and to suggest ways to reduce occu- pational exposure after high energy treatments. The findings may have clinical significance for cancer centers when reviewing protocols or operational proce- dures.}

\section{Methods}

\section{Ethics approval}

This study received ethics approval from the Department Research Committee at The Hong Kong Polytechnic University, and a letter of support from Tuen Mun Hospital (TMH).

\section{Treatment approaches}

Colorectal cancer is the third leading cancer killer in Hong Kong ${ }^{31)}$, and most patients would be advised to receive conventional pelvic irradiation as treatment. In some cases, to account for the large body separation at the pelvic region of patients, higher energy photon beams would be used for treatments ${ }^{30}$. In this study, three treatment approaches were assessed: $18 \mathrm{MV}$ and $10 \mathrm{MV}$ posterior-anterior (PA) opposed pelvic irradiations and a mixed energy, three-field three dimensional conformal technique (3DCRT) incorporating a $10 \mathrm{MV}$ posterior field and two $18 \mathrm{MV}$ lateral fields. Three treatment plans were delivered to a tissue equivalent pelvic phantom with a separation of $22 \mathrm{~cm}$. In order to resemble a patient with a body separation of $25 \mathrm{~cm}, 3 \mathrm{~cm}$ of the tissue equivalent Superflab ® bolus material was added to the posterior aspect of the phantom.

For the PA opposed treatment approach, a typical field size of $14 \mathrm{~cm} \times 14 \mathrm{~cm}$ was used, with markings on the pelvic phantom for reproducibility purposes. A dose prescription of 2 Gy per fraction was given as the central dose (CD). For each fraction, 456 monitor units (MUs) and 432 MUs were delivered with $10 \mathrm{MV}$ and $18 \mathrm{MV}$ photon treatments, respectively.
The conventional treatment plan was delivered to the pelvic phantom according to a mixed energy 3 -field Pinnacle v8.0m plan with 50 Gy prescribed to $97 \%$ of the point dose at the prescription point. The lateral fields received $18 \mathrm{MV}$ photon beams with 60-degree wedges, and a $10 \mathrm{MV}$ photon beam was used for the posterior field. The conventional plan was over 25 fractions, with 523 MUs per fraction.

\section{The radiotherapy machine unit at TMH, Hong Kong}

The study was conducted using the Elekta Precise Treatment System, which is capable of producing 6, 10 and18 MV photons. With MLCs, an electronic portal imaging system and a cone beam computed tomography system, this linear accelerator was suitable for all techniques ${ }^{32)}$. It is acknowledged as a limitation of this study that quantitative description on energy responses would help to explain the findings, and this is a consideration for further study, which will clarify the effects of individual activation products.

\section{Radiation monitoring devices}

The Mini 900R dose rate meter was designed for monitoring high level equivalent dose rates, scaling a broad range from 0.5 to $5,000 \mu \mathrm{Svh}^{-1}$ (0.05 to $500 \mathrm{mR} / \mathrm{h}$ ) and responding dominantly to X-ray and gamma radiations. Dose rate readings were provided every $8 \mathrm{sec}$. As it was mounted with an energy compensated Geiger-Mueller probe, it was capable of providing useful energy responses from $45 \mathrm{kV}$ to1.5 MV and above. The mean energies of radionuclides commonly induced by neutron interaction have been found to be $122 \mathrm{keV}, 480 \mathrm{keV}$ and $1.778 \mathrm{MeV}$ for ${ }^{57} \mathrm{Co},{ }^{187} \mathrm{~W}$ and ${ }^{28} \mathrm{Al}$, respectively ${ }^{33)}$. The radioactivity induced by interactions between neutrons and materials during high MV treatments fall within the dose range covered by this dose rate meter ${ }^{23,33,34)}$. The Mini 900R dose rate meter was thus suitable for monitoring the occupational exposure from the induced activity. Calibration was done using a ${ }^{137} \mathrm{Cs}$ source, and tests for linearity of response and sensitivity were conducted by medical physicists.

\section{Measurements with radiation monitoring devices}

Measurements were made at positions occupied by radiation therapists: at a fixed location in the treatment room $70 \mathrm{~cm}$ left lateral and $100 \mathrm{~cm}$ posterior to the isocenter at $100 \mathrm{~cm}$ above the floor (marked as location A in Fig. 1) and at the console control panel $\left(\right.$ marked as location B in Fig. 1) ${ }^{1)}$. Location A represented the therapists' typical working position ${ }^{24,34,35)}$, while the detector at location B checked the isolation of the accelerator bunker and detected any radiation leakage $^{3)}$. 


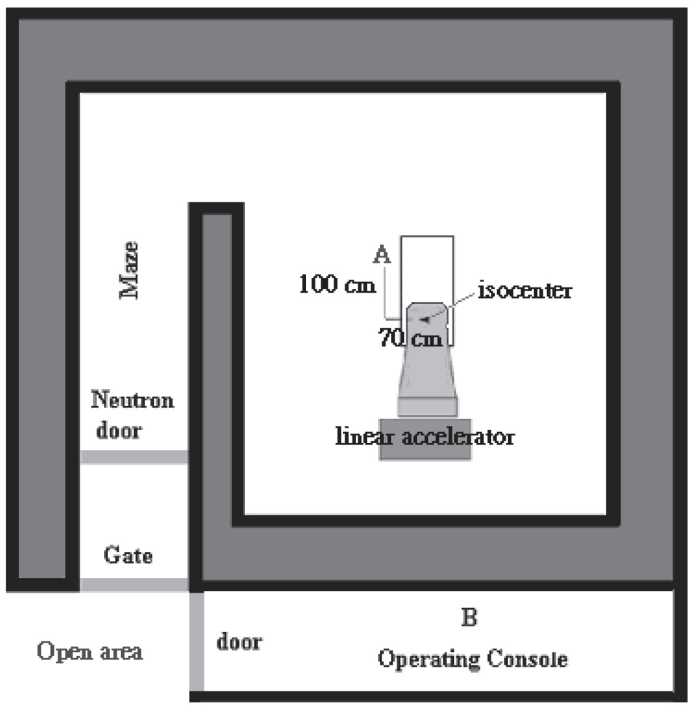

Fig. 1. The floor plan of the treatment room at Tuen Mun Hospital, indicating position of the detectors.

Measurements were taken simultaneously at the two locations during the treatment and repeated five times for the three techniques. The measurements of the dose rates commenced $30 \mathrm{sec}$ after the beam had stopped, representing the time required for radiation therapists to enter the treatment room after beam-off ${ }^{24)}$. The dose rate was measured with the Mini 900R dose rate meter and recorded at intervals of $30 \mathrm{sec}$ until the $15^{\text {th }}$ min. After each data recording, a time delay of 10 min elapsed before the start of the next experiment to allow for clearance of long-lived radioisotopes and simulation of the time interval between treatments in a realistic situation. This minimized the influence of the previous treatments due to accumulation of radioactivity ${ }^{3)}$. Elimination of the background radiation dose ensured that all results were due to radioactivity induced during the high-energy photon treatments.

\section{Determination of optimum room entry times}

In this study, determination of optimum room entry times was an important factor. In terms of radiation safety, three key factors were considered in order to calculate the optimum room entry times. These were equivalent dose, department workload and patient comfort. A balance was sought between the needs of patients and efficiency of service. Equivalent dose would be affected by exposure to radiation, so time spent in the treatment unit was balanced against the need to ensure reasonable patient throughput. Measurements were taken to calculate whether significant differences in measured equivalent doses could ensue as a consequence of delayed entry time, and consideration was given to how this would affect workload.

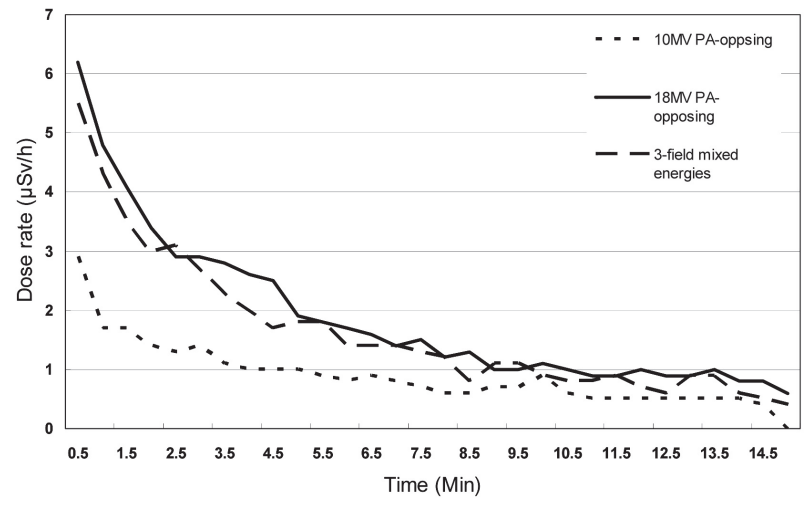

Fig. 2. Graph showing the dose rate at location A verses time after beam-off.

Statistical analysis

Measured equivalent doses were normally distributed. A two-way analysis of variance test was used to assess the differences between treatment approaches. The first factor was the treatment approach, and there were three levels, the 3-field 3DCRT approach and the $18 \mathrm{MV}$ and $10 \mathrm{MV}$ PA opposed techniques. The second factor was time, and there were several time intervals ranging from $0.5,1 \ldots 15 \mathrm{~min}$. Each subject was measured five times. The dependent measure was the measured equivalent dose of radiation therapists after a high-energy photon treatment fraction. The interaction effect was evaluated. One -way analysis of variance tests were performed individually for each treatment approach.

\section{Results}

Measurements of background levels and dose rates for each treatment approach

The background radiation levels were $0 \mu \mathrm{Sv} / \mathrm{h}$ at locations $\mathrm{A}$ and $\mathrm{B}$, so consequently, all further measurements were assumed to indicate the actual dose rates. The relationships between dose rates measured for each approach and time after beamoff were evaluated. The highest recorded dose-rate, $7 \mu \mathrm{Sv} / \mathrm{h}$, corresponded to the use of the $18 \mathrm{MV}$ PA opposed approach (Fig. 2). The maximum dose rates for the mixed-energy 3DCRT and $10 \mathrm{MV}$ PA opposed approaches were lower, at $5 \mu \mathrm{Sv} / \mathrm{h}$ and $3 \mu \mathrm{Sv} / \mathrm{h}$, respectively. For all three approaches, the dose rates decreased with time after beam-off. However, radiation levels remained measurable, even after beamoff at $15 \mathrm{~min}$, for all approaches. For the $10 \mathrm{MV}$ PA opposed approach, the radiation level was close to the background level at the $15^{\text {th }} \mathrm{min}$, while for the $18 \mathrm{MV}$ opposed approach, it remained at approximately $0.5 \mu \mathrm{Sv} / \mathrm{h}$.

The neutron dose rates at location $\mathrm{B}$ were close to $0 \mu \mathrm{Sv} / \mathrm{h}$ for all three approaches, indicating that the 


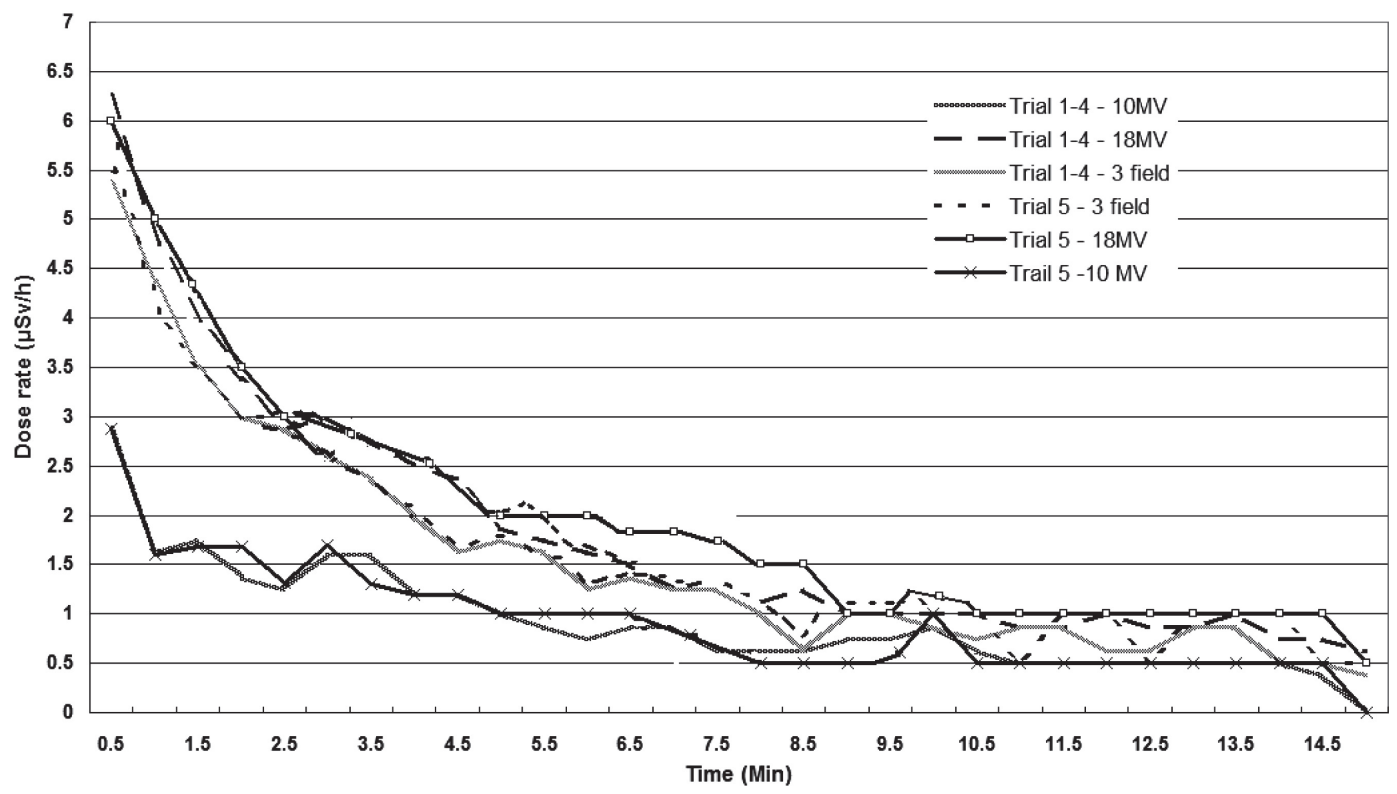

Fig. 3. Graph showing the relationship between average dose rates for the first 4 trials and the $5^{\text {th }}$ trial for all approaches.

occupational doses of radiation therapists were negligible.

When comparing dose rate measurements for the fifth trial (reading) with the average dose rates of the first four trials (readings), an accumulation effect was observed for all approaches. The dose rates of the fifth trial were higher than the average dose rates of the first four trials (Fig. 3).

\section{Interaction effect and optimum room entry times}

The interaction effect, i.e., the change in the simple main effect of treatment approaches over levels of time, was significant $(p<0.05)$. Furthermore, one-way ANOVA test indicated that the three treatment approaches were significantly different $(p<0.05)$. Post hoc analysis showed that differences in measured equivalent doses between the 3-field 3DCRT and $10 \mathrm{MV}$ PA opposed techniques and the differences in measured equivalent doses between the $18 \mathrm{MV}$ and $10 \mathrm{MV}$ PA opposed techniques were statistically significant $(p<0.05)$. In terms of safety limits, if one considers potential stochastic effects, which are independent of dose and have no threshold, then these findings are important regardless of the size of the differences concerned. In reality, only the first three time entry levels showed statistical significance when compared with further levels, i.e., time 0.5 to $1(p=0.001)$, time 1 to $1.5(p=0.012)$ and time 1.5 to $2(p=0.004)$, with later levels not proving significant $(p>0.05)$. Therefore, it was recommended to set $2 \mathrm{~min}$ as the safety limit and hence the minimum room entry time. However, this should not be equiva- lent to the optimum entry time.

According to the output of a one-way ANOVA test for the $18 \mathrm{MV}$ PA opposed technique, the dose rates at the $15^{\text {th }} \mathrm{min}$ were not statistically different from those at the $7^{\text {th }} \min (p>0.05)$. With a 7 -minute delayed entry time, the total treatment time would be $20.5 \mathrm{~min}$, and the patient throughput would be around 23 cases per day. Similarly, for the 3-field 3DCRT technique, the dose rates at the $15^{\text {th }} \mathrm{min}$ were not statistically different from those at $7.5 \mathrm{~min}$ $(p>0.05)$. With a 7.5-minute delayed entry time, the total treatment time would be $21.3 \mathrm{~min}$, and the patient throughput would be around 22 cases per day. Finally, for the $10 \mathrm{MV}$ PA opposed technique, the dose rates at the $15^{\text {th }}$ minute were not statistically different from those at the $11^{\text {th }} \min (p>0.05)$. With an 11-minute delayed entry time, the total treatment time would be $24.5 \mathrm{~min}$, and the patient throughput would be around 19 cases per day.

\section{Dose estimates for different radiation therapy regimes}

The average equivalent dose per year of a radiation therapist was calculated based on the dosimeter readouts. In the study, the model assumed the radiation therapists entered the treatment room at X min (where $X=0.5,1$ etc.) after beam-off and stayed in the treatment room for $10 \mathrm{~min}$. This working procedure was repeated for 200 working days per year. Hence, the annual occupational doses for radiation therapists would be

$\mathrm{D}_{\mathrm{r}} \times \mathrm{C} \times \mathrm{d}$, 
Table 1. Annual dose estimations based on the dose rate measurements

\begin{tabular}{|c|c|c|c|c|c|c|c|c|c|}
\hline \multirow{2}{*}{ Entry delay (min) } & \multicolumn{3}{|c|}{ No. of cases } & \multicolumn{3}{|c|}{ Cases/yr } & \multicolumn{3}{|c|}{ Annual dose (mSv/yr) } \\
\hline & 3-field & $18 \mathrm{MV}$ & $10 \mathrm{MV}$ & 3-field & $18 \mathrm{MV}$ & $10 \mathrm{MV}$ & 3-field & $18 \mathrm{MV}$ & $10 \mathrm{MV}$ \\
\hline 0.5 & 33 & 34 & 34 & 6,600 & 6,800 & 6,800 & 4.4 & 4.9 & 3.0 \\
\hline 1 & 32 & 33 & 33 & 6,400 & 6,600 & 6,600 & 4.3 & 4.7 & 2.9 \\
\hline 1.5 & 31 & 32 & 32 & 6,200 & 6,400 & 6,400 & 4.1 & 4.6 & 2.8 \\
\hline 2 & 30 & 30 & 30 & 6,000 & 6,000 & 6,000 & 4.0 & 4.3 & 2.6 \\
\hline 2.5 & 29 & 30 & 30 & 5,800 & 6,000 & 6,000 & 3.9 & 4.3 & 2.6 \\
\hline 3 & 28 & 29 & 29 & 5,600 & 5,800 & 5,800 & 3.7 & 4.1 & 2.5 \\
\hline 3.5 & 27 & 28 & 28 & 5,400 & 5,600 & 5,600 & 3.6 & 4.0 & 2.4 \\
\hline 4 & 26 & 27 & 27 & 5,200 & 5,400 & 5,400 & 3.5 & 3.9 & 2.3 \\
\hline 4.5 & 26 & 26 & 26 & 5,200 & 5,200 & 5,200 & 3.5 & 3.7 & 2.3 \\
\hline 5 & 25 & 25 & 25 & 5,000 & 5,000 & 5,000 & 3.3 & 3.6 & 2.2 \\
\hline 5.5 & 24 & 25 & 25 & 4,800 & 5,000 & 5,000 & 3.0 & 3.4 & 2.1 \\
\hline 6 & 24 & 24 & 24 & 4,800 & 4,800 & 4,800 & 2.9 & 3.1 & 1.9 \\
\hline 6.5 & 23 & 24 & 24 & 4,600 & 4,800 & 4,800 & 2.6 & 2.9 & 1.8 \\
\hline 7 & 23 & 23 & 23 & 4,600 & 4,600 & 4,600 & 2.5 & 2.7 & 1.6 \\
\hline 7.5 & 22 & 22 & 22 & 4,400 & 4,400 & 4,400 & 2.2 & 2.4 & 1.5 \\
\hline 8 & 21 & 22 & 22 & 4,200 & 4,400 & 4,400 & 2.0 & 2.2 & 1.4 \\
\hline 8.5 & 21 & 21 & 21 & 4,200 & 4,200 & 4,200 & 1.9 & 2.0 & 1.2 \\
\hline 9 & 21 & 21 & 21 & 4,200 & 4,200 & 4,200 & 1.7 & 1.9 & 1.2 \\
\hline 9.5 & 20 & 20 & 20 & 4,000 & 4,000 & 4,000 & 1.5 & 1.6 & 1.0 \\
\hline 10 & 20 & 20 & 20 & 4,000 & 4,000 & 4,000 & 1.4 & 1.5 & 1.0 \\
\hline 10.5 & 19 & 20 & 20 & 3,800 & 4,000 & 4,000 & 1.2 & 1.4 & 0.9 \\
\hline 11 & 19 & 19 & 19 & 3,800 & 3,800 & 3,800 & 1.1 & 1.2 & 0.8 \\
\hline 11.5 & 18 & 19 & 19 & 3,600 & 3,800 & 3,800 & 0.9 & 1.1 & 0.7 \\
\hline 12 & 18 & 18 & 18 & 3,600 & 3,600 & 3,600 & 0.8 & 0.9 & 0.6 \\
\hline 12.5 & 18 & 18 & 18 & 3,600 & 3,600 & 3,600 & 0.7 & 0.8 & 0.5 \\
\hline 13 & 17 & 18 & 18 & 3,400 & 3,600 & 3,600 & 0.6 & 0.6 & 0.4 \\
\hline 13.5 & 17 & 17 & 17 & 3,400 & 3,400 & 3,400 & 0.5 & 0.5 & 0.3 \\
\hline 14 & 17 & 17 & 17 & 3,400 & 3,400 & 3,400 & 0.4 & 0.4 & 0.3 \\
\hline 14.5 & 16 & 17 & 17 & 3,200 & 3,400 & 3,400 & 0.2 & 0.3 & 0.2 \\
\hline 15 & 16 & 16 & 16 & 3,200 & 3,200 & 3,200 & 0.1 & 0.1 & 0.1 \\
\hline
\end{tabular}

where $\mathrm{D}_{\mathrm{r}}$ is the sum of the dose rate in minutes at the console control panel during treatment and in the treatment room during patient set up. C represents the number of cases treated per day, and $\mathrm{d}$ indicates the 200 working days per year. The highest annual dose, corresponding to $4.9 \mathrm{mSv} / \mathrm{yr}$ (Table 1), was obtained when radiation therapists entered the treatment room immediately after an $18 \mathrm{MV}$ PA opposed treatment fraction. Therefore, the radiation therapists worked in the residual radiation area, and a sizeable radiation dose may be received.

\section{Discussion}

\section{Neutron production and activation}

Neutron production and material activation were observed for all treatment approaches. This mirrored similar studies, where neutron production occurred when high photon energies were used ${ }^{1,3,4,9,36)}$. According to Konefal et al., the energy threshold was about $8.5 \mathrm{MeV}$ for isotopes with a higher atomic number and about $10 \mathrm{MeV}$ for most light nuclei ${ }^{4)}$. For common materials comprising the components of the gantry, the thresholds of neutron production were $6-8 \mathrm{MeV}$ for lead and tungsten, $13 \mathrm{MeV}$ for aluminium and iron and $10 \mathrm{MeV}$ for copper ${ }^{1,9)}$. For higher-energy photon beams, it was easier to reach the threshold of neutron production for materials in the treatment room.

The neutron dose rate at location $\mathrm{B}$ was close to $0 \mu \mathrm{Sv} / \mathrm{h}$ for all three techniques. This agreed with a previous study in which the ambient dose equivalents in the console control area were often below the detection threshold ${ }^{1)}$. The results confirmed that the control area was sufficiently isolated and that negligible radiation leakage was detected.

The highest dose rate (approximately $7 \mu \mathrm{Sv} / \mathrm{h}$ ) corresponded to use of the $18 \mathrm{MV}$ PA opposed approach, whereas the maximum dose rates for the 
mixed-energies 3DCRT and $10 \mathrm{MV}$ PA opposed approaches were lower. At the outset of the study, it was anticipated that the 3 -field approach would produce higher dose rates, as wedged fields were used, and these have been reported to increase the production of secondary neutrons and hence increase the induced activity ${ }^{2}$. However, the results obtained were contrary to expectations. Two possible reasons account for this: firstly, delay in gantry rotations between fields interrupted the long irradiation time, and therefore, fewer neutrons were produced, reducing material activation ${ }^{34,37)}$. Also, the time delay allowed for decay of radioisotopes with short half-lives ${ }^{4}$. Secondly, photoneutron production during the $18 \mathrm{MV}$ PA opposed approach was higher than in the 3-field approach. Most radiation therapists start with the anterior field, i.e., the gantry at 180 degrees, to check for the possibility of any collision. As the treatment couch is a significant source of activation ${ }^{23,34)}$, the photonuclear reactions may be substantially increased.

For all three approaches, the dose rates decreased with time after beam-off. This may be due to ventilation that facilitated the air exchange rate in the treatment room, thus minimizing the doses from activation products in air $^{27)}$. One study has suggested that neutron capture leads to induced activity outside the treatment head, thus enhancing the contribution of the neutron-generated isotopes to staff dose, as these isotopes might be distributed throughout the treatment room. Clearly this raises potential radiation protection issues for staff working in medical linear accelerators $^{38)}$. Also, the dose from the decay of radioisotopes with short half-lives becomes immeasurable, as radioactive decay is exponential. The radiation levels remained measurable for all approaches 15 min after irradiation. As the delayed entry times after highenergy treatments in Hong Kong were not standardized, the potential exposure to staff should not be ignored.

\section{Accumulation effect}

An accumulation effect was clearly demonstrated for all three treatment approaches when comparing dose rate measurements of the fifth trial with the average dose rates of the first four trials (Fig. 3). Although a ten-minute period was allowed between each beam-on, radioisotopes with medium half-lives could still accumulate ${ }^{3)}$. As described by Rawlinson et al., ${ }^{56} \mathrm{Mn}$ and ${ }^{24} \mathrm{Na}$ attained radioactive equilibrium with increasing patient fractions ${ }^{30)}$. The build-up of radioactivity resulted in significant increase in occupational exposure when radiation therapists handled patients inside the treatment rooms ${ }^{4,34)}$. Also, consecutive treatments would lead to a more severe accumulation effect. Therefore, high-energy photon treatments should be spaced to avoid prolonged irradiation ${ }^{4)}$. Alternation between electron therapies and low-energy photon treatments enabled time for radioactive decay). Moreover, high-energy photon treatments could be scheduled at the latter part of the working day, so as to allow overnight decay of long-lived radioisotopes ${ }^{34}$.

\section{Variations in equivalent dose after different treatment techniques}

The differences in equivalent dose between the 3-field 3DCRT and $10 \mathrm{MV}$ PA opposed techniques and differences in equivalent dose between the $18 \mathrm{MV}$ and $10 \mathrm{MV}$ PA opposed techniques were statistically significant. The $10 \mathrm{MV}$ PA opposed technique produced relatively less neutrons. This finding suggested that radiation therapists should take the occupational dose as an additional consideration when deciding on treatment approach, especially if different treatment approaches can provide similar therapeutic effects for patients. In fact, one study has suggested that some treatment approaches should be avoided whenever possible ${ }^{34)}$. Research has also suggested that neutron doses outside the treatment field of an IMRT treatment increase by a factor of two $^{18)}$. Therefore, IMRT treatments should be delivered at X-ray energies lower than $18 \mathrm{MV}$ to reduce occupational hazards contributed by the neutron dose and activation dose ${ }^{34)}$.

\section{Dose rates versus time}

Only the first three time intervals showed any statistical significance when compared with further intervals, i.e., 0.5 to 1,1 to 1.5 and 1.5 to $2 \mathrm{~min}$ (Fig. 2). This implied that the dose rates and measured equivalent doses were significantly reduced if the radiation therapists waited for $2 \mathrm{~min}$ before entering the treatment room. However, it is recommended that radiation therapists wait for as long as possible. This conclusion can be explained by the fact that radiation will never fall to zero, and there are always minute amounts of residual radiation in the treatment room. The linear dose-response model of radiation suggests that any increase in dose, no matter how small, results in an increase in risk. Studies of occupational workers exposed to chronic low levels of radiation, above normal background, have shown higher probability with regards to developing leukemia and other cancers $^{39)}$.

According to annual dose estimations, $3.0 \mathrm{mSv} / \mathrm{yr}$ would be received if radiation therapists entered the treatment room immediately after beam-off for a $10 \mathrm{MV}$ PA opposed treatment (Table 1). Although the estimated equivalent doses were lower than the maximum permissible dose values ${ }^{21)}$, exposure due to induced activation cannot be neglected, and it is important to investigate dose reduction strategies 
when using high-energy photon beams. The linear no-threshold (LNT) model works on the principle that if the radiation dose is negligible, then the risk is also negligible. However, quantitative analysis on the LNT model fell outside the limits of this study and was not evaluated.

The highest annual dose, corresponding to $4.9 \mathrm{mSv} / \mathrm{yr}$ (Table 1), was obtained when radiation therapists entered the treatment room immediately after an $18 \mathrm{MV}$ PA opposed treatment. The results concurred with published estimates of the annual activation dose received by staff during routine procedures, which were in the range of $0.7-5 \mathrm{mSv} / \mathrm{yr}^{27,40,41)}$. Even though estimated annual doses for all approaches were below maximum permissible dose values, i.e., $20 \mathrm{mSv} / \mathrm{yr}^{20)}$, radiation therapists should remain alert to protect themselves from unnecessary radiation and limit the equivalent dose to as low as reasonably achievable, especially since the thermoluminescent dosimeter records for most staff at $\mathrm{TMH}$ tend to be approximately $2 \mathrm{mSv} / \mathrm{yr}$.

\section{Dose reduction strategies}

1) Optimum room entry times

Findings indicated that radiation therapists should wait at least 2 min before entering the treatment room. Even though the highest estimated annual dose was well below the maximum permissible dose value of $20 \mathrm{mSv} / \mathrm{yr}$, it is still important to reduce the absorbed dose as much as possible so as to lower corresponding risk $^{39)}$. However, it is impractical to wait for too long in the clinical situation, as there is a waiting list of patients and tolerance of patients should be considered in terms of delay. The optimum entry time should be a balance between maximum dose reduction, patient comfort and treatment efficiency.

The results indicated that the delay time for the 10 MV PA opposed technique was longer than for the other two techniques, that is, $11 \mathrm{~min}$. This may be because the dose rates fluctuated and remained relatively high, and so statistical differences were detected even $10 \mathrm{~min}$ after beam-off. However, all suggested delay times gave a reasonable treatment time but a significant reduction in exposure to the radiation therapists. The results may be useful for cancer centers when considering protocols or operational procedures.

To facilitate treatment efficiency, special arrangements should be made. For example, mixed energy 3-field 3DCRT has two $18 \mathrm{MV}$ lateral fields. These could be arranged as the first two fields to allow for radioactivity to decay during the third field, thus reducing the delay time after treatments. Moreover, radiation therapists should make use of the delay time for preparation of the next treatment. For example, they could manually manipulate the gantry and the collimators, thereby facilitating the treatment workflow and compensating for longer treatment times.

2) Good working habits and procedures

A correlation exists between risks and personnel training, workload and operational procedures ${ }^{24)}$. Therefore, radiation therapists should be alert and skilled in operational procedures to avoid accidental exposure. Wearing neutron dosimeters can help monitor the personal dose of radiation therapists who work in residual radiation fields ${ }^{42}$. Special arrangements could be made to concentrate high-energy treatments in the afternoon sessions or on Fridays. This effectively minimized the occupational dose due to medium- and long-lived isotopes and allowed time for decay over the weekends ${ }^{24)}$.

3) Dose reduction strategies regarding treatment room and machine design

To further reduce induced radioactivity, one could avoid using materials that can be activated as treatment accessories ${ }^{34)}$; use neutron moderating and absorption materials in the walls of the accelerator maze, e.g., flex boron and polyethylene ${ }^{43-45)}$; stop neutrons from escaping by closure of MLCs and jaws after treatments ${ }^{1)}$; and install additional multileaf shielding systems to reduce neutron exposure without interfering with the therapeutic beam ${ }^{19}$.

\section{Conclusions}

Although the potential exposure risks for radiation therapists fell well within internationally recommended values, the results indicated that risks can be further decreased by delaying room entry times. It was suggested that $7 \mathrm{~min}$ was the optimum room entry time for $18 \mathrm{MV}$ PA opposed techniques, $7.5 \mathrm{~min}$ was the optimum entry time for 3-field 3DCRT treatments and $11 \mathrm{~min}$ was the optimum entry time for 10 MV PA opposed techniques. The suggested delay times gave moderate treatment times but a significant reduction in dose to radiation therapists.

Acknowledgments: This work was supported by the Clinical Oncology Department and Medical Physics Department of Tuen Mun Hospital.

\section{References}

1) Donadille L, Trompier F, Robbes I, et al. Radiation protection of workers associated with secondary neutrons produced by medical linear accelerators. Radiation Measurements 2008; 43: 939-43.

2) Mesbahi A, Keshtkar A, Mohammadi E, Mohammadzadeh M. Effect of wedge filter and field size on photoneutron dose equivalent for an $18 \mathrm{MV}$ photon beam of a medical linear accelerator. Appl Radiat Isot 2010; 68: 84-9. 
3) Konefal A, Polaczek-Grelik K, Zipper W. Undesirable nuclear reactions and induced radioactivity as a result of the use of the high-energy therapeutic beams generated by medical linacs. Radiat Prot Dosimetry 2008; 128: 133-45.

4) Konefal A, Orlef A, Dybek M, Maniakowski Z, Polaczek-Grelik K, Zipper W. Correlation between radioactivity induced inside the treatment room and the undesirable thermal/resonance neutron radiation produced by linac. Physica Medica 2008; 24: 212-8.

5) National Council on Radiation Protection and Measurements (NCRP), Report No. 79, Neutron Contamination from Medical Electron Accelerators. Bethesda (MD): 1984.

6) Ongaro C, Zanini A, Nastasi U, Ródenas J, Ottaviano G, Manfredotti C. Analysis of photoneutron spectra produced in medical accelerators. Phys Med Biol 2000; 45: 55-61.

7) Lin JP, Liu WC, Lin CC. Investigation of photoneutron dose equivalent from the high-energy photons in radiotherapy. Appl Radiat Isot 2007; 65: 599-604.

8) Kry SF, Salehpour M, Followill DS, et al. Out-of field photon and neutron dose equivalents from stepand-shoot intensity- modulated radiation therapy. Int J Radiat Oncol Biol Phys 2005; 62: 1204-16.

9) Hall EJ, Martin SG, Howard A, Hei TK. Photoneutrons from medical linear accelerators radiobiological measurements and risk estimates. Int J Radiat Oncol Biol Phys 1995; 33: 225-30.

10) Kilagua P, Howard A. Photoneutrons from high energy medical linear accelerators measurement of the spectrum and dose using a miniature proportional counter. Int J Radiat Oncol Biol Phys 1995; 31: 629-33.

11) McGinley PH, Landry JC. Neutron contamination of X-ray beams produced by the Varian Clinac 1800 . Phys Med Biol 1989; 34: 777-83.

12) Barquero $R$, Mendez $R$, Vega-Carrillo $H R$, Iniguez MP, Edwards TM. Neutron spectra and dosimetric features around an $18 \mathrm{MV}$ linac accelerator. Health Phys 2005; 88: 48-58.

13) Followill DS, Stovall MS, Kry SF, Ibbott GS. Neutron source strength measurements for Varian, Siemens, Elekta, and General Electric linear accelerators. J Appl Clin Med Phys 2003; 4: 189-94.

14) Kry SF, Ponisch F, Vassiliev ON, Salepour M, Gillin M, Mohan R. Reduced neutron production through the use of flattening -filter-free Accelerator. Int J Radiat Onco Biol Phys 2007; 68: 1260-4.

15) Hashemi SM, Hashemi-Malayeri B, Raisali G, Shokrani P, Sharafi AA, Jafarizadeh M. The effect of field modifier blocks on the fast photoneutron dose equivalent from two high-energy medical linear accelerators. Radiat Prot Dosimetry 2008; 128: 359-62.

16) Silari M. Workplace characterization in mixed neutron-gamma fields, specific requirements and available methods at high-energy accelerators. Radiat Prot Dosimetry 2007; 124: 230-44.

17) Howell RM, Kry SF, Burgett E, Followill D, Hertel
NE. Effects of tertiary MLC configuration on secondary neutron spectra from $18 \mathrm{MV}$ x-ray beams for the Varian 21EX linear accelerator. Med Phys 2009; 36: 4039-46.

18) Ipe NE, Roesler S, Jiang SB, Ma CM. Neutron measurement for intensity modulated radiation therapy. Engineering in Medicine and Biology Society, 2000. Proceedings of the 22nd Annual International Conference of the IEEE 2000; 4: 3234-7.

19) RebelloWF, Silva AX, Facure A. Multileaf shielding design against neutrons produced by medical linear accelerators. Radiat Prot Dosimetry 2008; 128: 227-33.

20) Sohrabi M, Mostofizadeh A. Measurement of photoneutron doses in and out of high energy x-ray beam of a Saturne-20 medical linear accelerator by ECE Polycarbonate detectors. Radiation Measurements 1999; 31: 479-82.

21) International Commission on Radiological Protection. Recommendation of the International Commission on Radiological Protection. Oxford: Pergamon Press; ICRP Publication 60, Ann ICRP p.106-42; 1991.

22) National Council on Radiation Protection and Measurements. Limitation of exposure to ionizing radiation. NCRP Report 116. Bethesda (MD): National Council on Radiation Protection and Measurements; 1993.

23) Fischer HW, Tabot B, Poppe B. Comparison of activation products and induced dose rates in different high-energy medical linear accelerators. Health Phys 2008; 94: 272-8.

24) Grundel M, Guthoff F. Radiation burden of assistant medical technicians at a medical accelerator. J Med Phys 2008; 33: 171-4.

25) McGinley PH. Dose to radiotherapy technologist from air activation. Med Phys 1984; 14: 855-8.

26) McGinley PH. Air activation produced by highenergy medical accelerators. Med Phys 1983; 10 : 796-800.

27) Almen A, Ahlgren L, Mattsson S. Absorbed dose to technicians due to induced activity in linear accelerators for radiation therapy. Phys Med Biol 1991; 36: 815-22.

28) Fischer HW, Peick K. Activation products in a medical linear accelerator. Radiotherapy Oncol 1999; 51 (Suppl. 1): S59.

29) Saeed MK, Moustafa O, Yasin OA, Tuniz C, Habbani FI. Dose to patients from photoneutrons emitted in a medical linear accelerator. Int J Radiat Oncol Biol Phys 2005: 66: 1060-73.

30) Rawlinson JA, Islam MK, Galbraith DM. Dose to radiation therapists from activation at high-energy accelerators used for conventional and intensitymodulated radiation therapy. Med Phys 2002; 29: 598-608.

31) Hospital Authority. Leading Cancer Sites in Hong Kong in 2007. [Online]. 2007 [cited 2010 Apr 7]; Available from: URL: http://www3.ha.org.hk/ cancereg/e_rank.pdf 
32) La Riviere PD. Radiotherapy technologist dose from high-energy medical accelerators. Health Phys 1985; 49: 1105-14.

33) Tofani A, Del CA, Manetti F. Assessment of potential exposure risk for radiotherapy staff working with lineal accelerators. Radiol Med 1999; 97: 286-95.

34) Reft CS, Alecu R, Das IJ, et al. Dosimetric considerations for patients with HIP prostheses undergoing pelvic irradiation. Report of the AAPM Radiation Therapy Committee Task Group 63. Med Phys 2003; 30: 1162-82.

35) Precise treatment system. [Online]. 2008 [cited 2010 Apr 1]; Available from: URL: http://www.elekta. com/assets/Elekta-Oncology/Precision-RadiationTherapy/brochures/Precise-Treatment-Systembrochure-0185-10-08.pdf

36) Kim KY, Kwak JW, Park SH, et al. Identification of Neutron-Induced Isotopes in Varian LINAC System. IFMBE Proceedings 2009; 25: 616-9.

37) Domingo C, García-Fusté MJ, Morales E, et al. Neutron spectrometry and determination of neutron ambient doses in radiotherapy treatments under different exposure conditions. IFMBE Proceedings 2009; 25: 523-6.

38) Fischer HW, Tabot BE, Poppe B. Activation processes in a medical linear accelerator and spatial distribution of activation products. Phys Med Biol
2006; 51: 461-6.

39) Garnica-Garza HM. Characteristics of the photoneutron contamination present in a high-energy radiotherapy treatment room. Phys Med Biol 2005; 50: 531-9.

40) Cardis E, Vrijheid M, Blettner M, et al. Risk of cancer after low doses of ionising radiation: retrospective cohort study in 15 countries. BMJ 2005; 331: 77-80.

41) McGinley PH, Butker EK. Evaluation of neutron dose equivalent levels at the maze entrance of medical accelerator treatment rooms. Med Phys 1991; 18: 279-81.

42) Powell NL, Newing A, Bullen MA, Sims C, Leaton SF. A radiation safety survey on a Clinac-20 linear accelerator. Phys Med Biol 1987; 32: 707-18.

43) O'Brien P, Michaels HB, Gillies B, Aldrich JE, Andrew JW. Radiation protection aspects of a new high-energy linear accelerator. Med Phys 1985; 12: 101-7.

44) Kersey RW. Estimation of neutron and gamma radiation doses in the entrance mazes of SL75-20 linear accelerator treatment rooms. Med Mundi 1979; 24: 151-5.

45) Falca RC, Facure A, Silva AX. Neutron dose calculation at the maze entrance of medical linear accelerator rooms. Radiat Prot Dosimetry 2007; 123: 283-7. 